\title{
Is the prevalence of Klinefelter syndrome increasing?
}

\author{
Joan K Morris ${ }^{\star 1}$, Eva Alberman ${ }^{1}$, Claire Scott $^{2}$ and Patricia Jacobs ${ }^{3}$
}

\begin{abstract}
${ }^{1}$ Centre for Environmental and Preventive Medicine, Wolfson Institute of Preventive Medicine, Barts and the London, Queen Mary's School of Medicine and Dentistry, Charterhouse Square, London EC1M 6BQ, UK; ${ }^{2}$ Chromosome Abnormality Database, Oxford Genetics Laboratories, Churchill Hospital, Oxford OX3 7LJ, UK; ${ }^{3}$ Division of Human Genetics, University of Southampton Medical School, Wessex Regional Genetics Laboratory, Salisbury District Hospital, Salisbury SP2 8BJ, UK
\end{abstract}

The birth prevalence of sex chromosome trisomies (SCT), that is individuals with an XYY, XXY or XXX sex chromosome constitution, is traditionally based on six surveys of unselected newborns carried out in the 1960 s and early 1970s. All three SCTs had a prevalence of 1 in 1000 same sex births. We re-examined these prevalences based on additional cytogenetic studies of newborn surveys, spontaneous abortions, perinatal deaths and prenatal diagnoses. The more recent newborn surveys suggest there has been an increase in the prevalence of $X X Y s$, but not of the other two SCTs since the original newborn series. The prevalence of $X X Y s$ has risen from 1.09 to 1.72 per 1000 male births $(P=\mathbf{0 . 0 2 3})$. We suggest that such an increase, in the absence of an increase in the prevalence of $X X X$, is unlikely to be due to increased maternal age. As XXY is the only chromosome abnormality known where a substantial proportion $(\sim 50 \%)$ arise as the result of non-disjunction at the first paternal meiotic division, we speculate that some factor may be interfering with pairing and/or recombination of the sex bivalent at the paternal MI division.

European Journal of Human Genetics (2008) 16, 163-170; doi:10.1038/sj.ejhg.5201956; published online 14 November 2007

Keywords: Klinefelter syndrome; prevalence; sex chromosome trisomies

\section{Introduction}

Traditionally, information on the prevalence of sex chromosome trisomies is based on six large surveys of consecutive newborns carried out in the late 1960s and early 1970 s (summarised by Hook and Hamerton in 1977). ${ }^{1}$ All three sex chromosome trisomies had a similar live birth prevalence of 1 per 1000 same sex births. However, recent data from the UK Chromosome Abnormality Database showed a much greater number of prenatal diagnosis of XXY (Klinefelter's syndrome) than either XXX (Triple X) or XYY (unpublished communication, Claire Scott). The

*Correspondence: Dr JK Morris, Centre for Environmental and Preventive Medicine, Wolfson Institute of Preventive Medicine, Barts and the London, Queen Mary's School of Medicine and Dentistry, Charterhouse Square, London EC1M 6BQ, UK.

Tel: + 44207882 6274; Fax: + 442078826270 ;

E-mail: j.k.morris@qmul.ac.uk

Received 28 June 2007; revised 4 October 2007; accepted 10 October 2007; published online 14 November 2007 excess of XXY prenatal diagnoses could be explained either by much greater fetal losses occurring in XXY, than in XXX or XYY pregnancies between the time of prenatal diagnosis and birth or by an increase in the prevalence, possibly due to the increases in maternal age that have occurred since the 1970s. If the latter were the case this should also apply to XXX pregnancies, which like XXY pregnancies increase in prevalence with maternal age. In this paper, we collate the evidence on the prevalence of XYY, XXY and XXX from studies on series of newborns, spontaneous fetal and perinatal deaths and prenatal diagnoses to determine an explanation for the greater numbers of XXY prenatal diagnoses observed.

Materials and methods

Data sets

Newborn series Sixteen published series of consecutive liveborns in which chromosome banding was used were 
identified using MEDLINE (Supplementary Table 1). ${ }^{2-17}$ Six studies started to collect data prior to 1971 and covered the period 1967-1974. ${ }^{2-7}$ Their results were summarised by Hook and Hammerton $(1977){ }^{1}$ we will refer to these as the early newborn studies. These studies are compared to the 10 remaining studies that collected data from 1971 to 1988; referred to as the late newborn studies. ${ }^{8-17}$ The details are given in Supplementary Table 1 and summarised in Table 1. Nielsen et al collected two series of data from consecutive liveborns from 1969 to 1974 and from 1980 to 1988. Hook and Hammerton included the series from 1969 to 1974 in their summary and it is therefore classified as an early newborn study. The series from 1980 to 1988 is classified as a late newborn study.

Spontaneous abortions and perinatal deaths Twelve informative published series of spontaneous abortions in which chromosome banding was used were identified. ${ }^{18-29}$ A small number of other such studies were not included because only autosomal trisomies were specified, and sex trisomies would have been grouped as 'other' anomalies. Unpublished data from Warburton, Hassold and Ljunger augmented these published data.

The series (Table 1 and Supplementary Tables 2 and 3) were grouped into two sets. One was of products of conception, naturally expelled or delivered by dilatation and curettage, that were cultured for cytogenetic examination. ${ }^{18-23}$ The other was where the chromosomes of the chorionic villi $(\mathrm{CV})$, naturally expelled or obtained by trans-abdominal or cervical sampling, were examined directly without an intervening culture period. ${ }^{24-29}$ The cultured series were mostly derived from surveys of women attending hospital because of threatened or completed abortion. These included pregnancies of later gestational age and younger maternal age than the direct chorionic villi series. The latter were mostly derived from women in whom a first trimester prenatal ultrasound examination, sometimes as a part of a formal prenatal screening test for Down's syndrome, had confirmed a fetal death. The cultured series tended to predate the direct villi series.

The data on perinatal deaths in which the chromosomes had been banded is very sparse and consists of only four published surveys (Table 1 and Supplementary Table 4). ${ }^{30-32}$

Prenatal diagnoses We considered two large series from the early 1980s giving the results of fetal amniocentesis performed solely on the grounds that the mother was 35 years of age or over and therefore at an increased risk of Down's syndrome. ${ }^{33,34}$ These give us an unbiased picture about the prevalence of different chromosome anomalies at 15-17 weeks of gestation but only for mothers of 35 years of age or older (Table 1 and Supplementary Table 5).

More representative data are available from the UK Chromosome Abnormality Database (CAD). ${ }^{35}$ Since January 1980 CAD has collected data from the majority of cytogenetic laboratories in the United Kingdom on chromosomal abnormalities they found in pre- and postnatal diagnoses. We examined the CAD prenatal diagnoses records, from January 1980 to May 2006 (Supplementary Table 5). These include information on age of mother, date of sample, sample used (amniocentesis vs CVS), fetal sex and indications for prenatal diagnosis. The prevalences of the sex chromosome trisomies cannot be estimated from the CAD data as there are no data on the total number of diagnostic tests that are performed.

The indications for prenatal diagnosis included (i) maternal age alone, (ii) the results of testing for Down's syndrome based on algorithms using maternal age and/or

Table 1 Prevalence 47, XYY, 47XXY and 47,XXX in studies of newborns, spontaneous abortions, prenatal diagnoses and perinatal deaths

\begin{tabular}{|c|c|c|c|c|c|c|c|}
\hline & \multirow{2}{*}{$\begin{array}{l}\text { Years of data } \\
\text { collection }\end{array}$} & \multicolumn{3}{|c|}{ Number of cases } & \multicolumn{3}{|c|}{ Prevalence $^{\mathrm{a}}$ per 1000 (95\% Cl) } \\
\hline & & $X Y Y$ & $X X Y$ & $X X X$ & $X Y Y$ & $X X Y$ & $X X X$ \\
\hline \multicolumn{8}{|l|}{ Newborn studies } \\
\hline Early $2-7$ & $1967-1971$ & 42 & 41 & $21^{\mathrm{b}}$ & $1.11(0.82-1.50)$ & $1.09(0.80-1.47)$ & $1.10(0.71-1.68)$ \\
\hline Late $^{8-17}$ & $1971-1988$ & 36 & 58 & 32 & $1.07(0.77-1.48)$ & $1.72(1.33-2.23)$ & $0.99(0.70-1.40)$ \\
\hline \multicolumn{8}{|l|}{ Spontaneous abortions } \\
\hline Culture ${ }^{18-23}$ & $1975-2005$ & 1 & 17 & 3 & $0.2(0.0-1.4)$ & $4.2(2.4-6.7)$ & $0.7(0.1-2.1)$ \\
\hline $\mathrm{CVS}^{24-29}$ & $1987-2005$ & 1 & 10 & 4 & $1.3(0.0-7.2)$ & $13.1(6.3-24.0)$ & $5.3(1.4-13.4)$ \\
\hline Perinatal deaths ${ }^{30-32}$ & & 3 & 3 & 1 & $4.6(0.9-13.4)$ & $4.6(0.9-13.4)$ & $1.5(0.0-8.5)$ \\
\hline \multicolumn{8}{|c|}{ Prenatal diagnoses series } \\
\hline \multirow{2}{*}{$\begin{array}{l}\text { Amniocentesis series } \\
\text { all women }>35^{33,34} \\
\text { CAD series }\end{array}$} & $1976-1981$ & 28 & 112 & 85 & $0.77(0.51-1.11)$ & $3.08(2.54-3.71)$ & $2.34(1.87-2.89)$ \\
\hline & $1980-2006$ & 225 & 542 & 350 & - & - & - \\
\hline
\end{tabular}

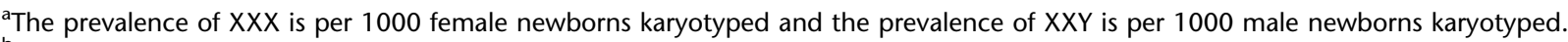

${ }^{b}$ The number of $X X X$ births is fewer than those of XYY and XXY due to one large study only examining male babies. 
serum screening and nuchal translucency screening and (iii) an abnormal ultrasound scan. Bias in the relative frequencies of the different sex chromosome trisomies may arise if, for example, XYY pregnancies were more likely to be found to have abnormal serum markers that are used in Down's syndrome serum screening than XXX or XYY pregnancies. However, there is no evidence that extreme levels of the screening markers or abnormal ultrasound scans are associated with an increased prevalence of any of the sex chromosome trisomies at 15-17 weeks of gestation. ${ }^{36,37}$

\section{Statistical methods}

The prevalence of XXX is always expressed as a proportion of all female pregnancies and the prevalence of XYY and $\mathrm{XXY}$ are always expressed as the proportion of all male pregnancies. In studies in which only the total number of pregnancies is given, the split between male and female fetuses is assumed to be 50:50.

Mosaics with a normal and a trisomic cell line are included in all the analyses, as there is evidence that the majority of such mosaics result from trisomic conceptions. ${ }^{38}$ All other mosaics have been excluded.

The live birth prevalence of XYY, XXY and XXX is analysed by fitting Poisson regression models to determine whether there is a difference in the prevalence of these anomalies in the early newborn studies compared to the later newborn studies. A random effects model is used to estimate the average live birth prevalence.

\section{Results}

\section{Newborn studies}

Figures 1-3 illustrate the observed livebirth prevalence from each of the studies for each of the sex chromosome trisomies split according to the earlier and later studies. Table 1 and Supplementary Table 1 summarize the overall prevalence. Fitting Poisson regression models showed no evidence of greater variance than expected between the studies. There has been a statistically significant increase in the prevalence for XXY of 59\% (95\% CI: 7-137\%; $P=0.023$ ). For the earlier studies, the prevalence was 1.09 per 1000 live births compared to 1.72 for the later studies. For XXX and $X Y Y$ there was no evidence that there has been an increase in birth prevalence ( $P=0.87$ and 0.73 respectively), with both having a prevalence of around 1.1 per 1000 births.

\section{Spontaneous abortions and perinatal deaths}

The prevalence of chromosomal anomalies in spontaneous abortions is strongly associated with the gestational age at karyotyping, since many chromosomal anomalies are lost before the pregnancy has been recognised and karyotyped.

As described in the Methods section, the cultured series were mostly derived from surveys of women attending hospital because of threatened or completed abortion. These included pregnancies of later gestational age and younger maternal age than the direct villi series, which were generally based on women in whom a first trimester ultrasound scan had confirmed a fetal death. The cultured series were more often contaminated with maternal cells and the abnormality rate in the later series was higher.

Nevertheless, the pattern of the findings regarding the sex chromosome trisomies was consistent (Table 1). XXYs are much more frequent than the other two sex chromosome trisomies, in both series, although their prevalence was highest in the first-trimester samples. XYY fetuses were uncommon in all studies; only two cases were observed. Therefore, the XXYs, and possibly the XXXs, are more likely to abort spontaneously early in gestation prior to prenatal diagnosis and also to be lost later after the time at which prenatal diagnosis occurs.

The perinatal deaths include three cases of XXY, one of $X X X$, and three of XYY. The numbers are very small and the unexpectedly large number of XYYs in this group is likely to be due to chance, although it may reflect a propensity of such males to die in the perinatal period.

\section{Prenatal diagnoses}

Supplementary Table 5 shows that in the two large prenatal diagnosis series (1977-1981), the prevalence of XXY is about 30\% higher than that of XXX (3.1 per 1000 compared to 2.3 per 1000). Both prevalences are higher than in the newborn series. This may be due to the prenatal diagnoses series being restricted only to women over 35 years of age and the fact that the risk of an XXX or XXY pregnancy increases with maternal age. This is consistent with the prevalence of XYY being the lowest, as maternal age is not associated with XYY.

Data from the Chromosome Abnormality Database (CAD) gives the number of prenatal diagnoses according to whether the samples were from an amniocentesis or chorionic villi (Supplementary Table 5). In the CVS and the amnio samples, there were about $50 \%$ more cases of XXY than of XXX. There were fewer cases of XYY observed than of the other sex chromosome trisomies, which is as expected due to the selection of mothers of high maternal age. The ratio of XXY to XXX cases diagnosed remained constant over the time of the register, but due to the small sample sizes, there is very low power to detect any changes. We further considered only the samples from women said to be tested only because of raised maternal age (data not shown), where there could be no bias due to selection by serum or ultrasound markers or an abnormal scan. The findings in this subgroup were similar to that of the total group.

\section{Prevalence of XYY, XXY and XXX in clinically recognised pregnancies}

Table 2 uses the live birth prevalence estimated from the later studies and the estimates from the spontaneous abortions and perinatal deaths, to work backwards to estimate the prevalence of the sex chromosome trisomies 


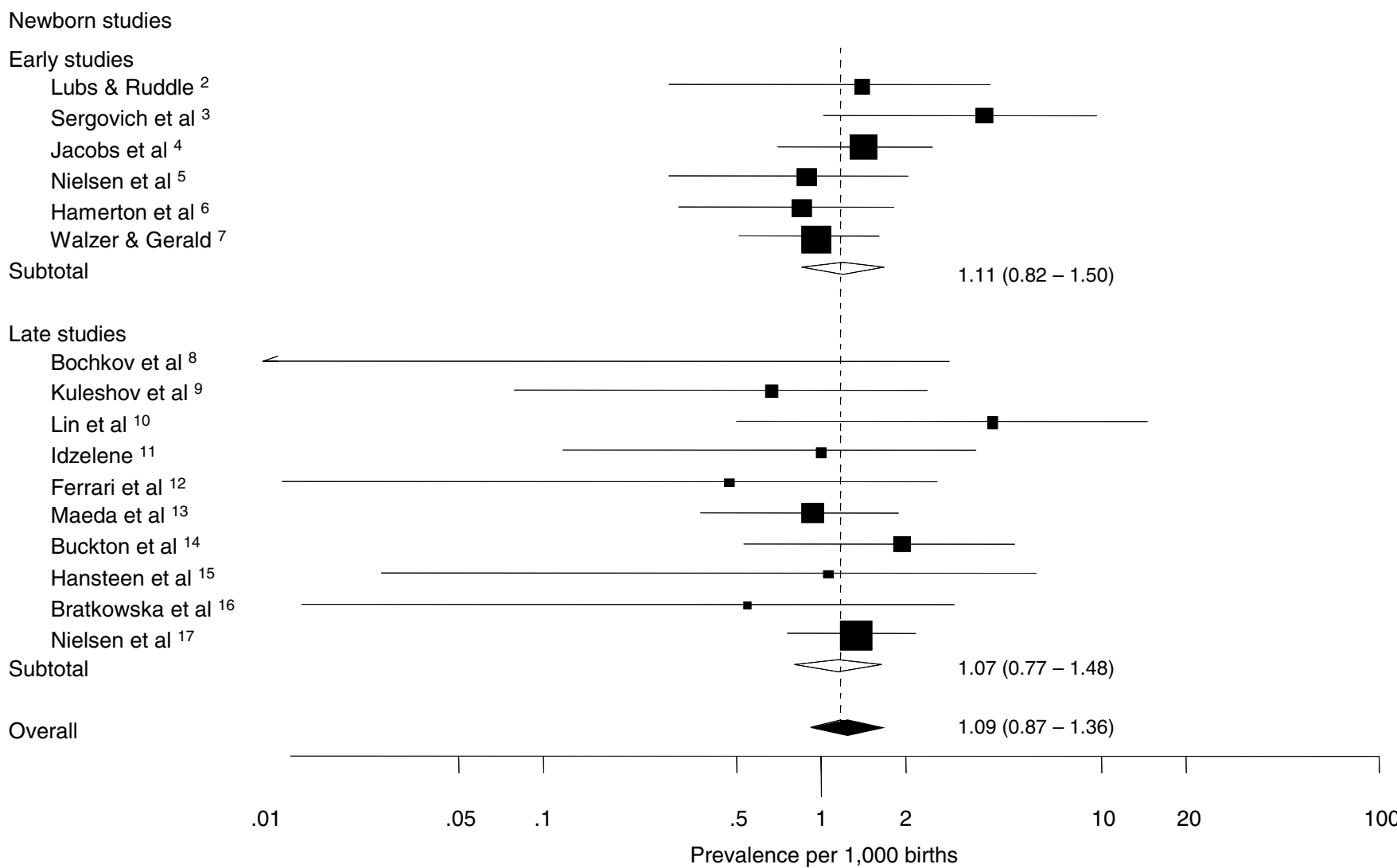

Figure 1 Live birth prevalence of XYY. Squares are estimates of prevalence sized according to the size of the study (studies with no affected babies have no square). Horizontal lines are the $95 \%$ confidence intervals of the prevalence in each study. Empty diamonds are summary estimates of the prevalence for the early and late studies. Full diamond is overall estimate of early and late series combined, with the vertical dashed line at the estimate. The size of the diamond indicates the $95 \% \mathrm{Cl}$.

in all clinically recognised pregnancies. It assumes that $20 \%$ of all recognised pregnancies will end in a spontaneous abortion, $1 \%$ in a perinatal death and $79 \%$ in a live birth. The prevalence for the spontaneous abortion series is calculated as $20 \%$ of the mean of the two types of spontaneous abortion series weighted according to the number of sex chromosome trisomies identified. For example for XXY 17 cases were identified in the cultured series with a prevalence of 4.2 per 1000 and 10 cases were identified in the direct villi series with a prevalence of 13.1 per 1000. The combined prevalence was calculated as $(17 \times 4.2+10 \times 13.1) /(17+10)=7.5$ per 1000 and $20 \%$ of this is 1.5 per 1000 (the figure in Table 2)

The prevalence in recognised pregnancies of $\mathrm{XXY}$ is twice as great as that of XXX (2.91 per 1000 vs 1.47 per $1000)$, both being much greater than that of XYY (1.05 per 1000 ). Only $59 \%$ of XXY and $67 \%$ of XXX pregnancies result in live birth but, due to the assumptions made to obtain these figures, it is not possible to test whether they are statistically, significantly different. However, they are both clearly different from the approximate 100\% of XYY pregnancies that result in live birth.

\section{Discussion}

The greater number of prenatal diagnoses of XXY than either XXX or XYY reported in the UK Chromosome Abnormality Database is consistent with their prevalence at birth in the later newborn surveys and the data on spontaneous abortions and perinatal deaths. A comparison of the later newborn surveys with the original newborn surveys carried out in the late 1960s and early 1970s demonstrated an increased birth prevalence of XXYs, but not of XYYs nor of XXXs. While the apparent increase in $\mathrm{XXY}$ might be the result of errors in the early new born surveys there is no plausible reason why the XXYs but not the XXXs or XYYs would have been underestimated. Furthermore, the prevalence of XXYs is consistent among the ten later surveys.

During the period of the early newborn series there were no prenatal diagnoses and thus no selective terminations of chromosome abnormalities. In contrast, during the period of the later newborn surveys prenatal diagnosis was introduced resulting in selective termination of some chromosomally abnormal fetuses. Data from the Wessex region between 1976 and 2005 indicates that XXYs were 
Newborn studies

Early studies
Lubs \& Ruddle ${ }^{2}$
Sergovich et al ${ }^{3}$
${\text { Jacobs et al }{ }^{4}}$ Nielsen et al $^{5}$
Hamerton et al ${ }^{6}$
Walzer \& Gerald ${ }^{7}$
Subtotal
Late studies
Bochkov et al ${ }^{8}$
Kuleshov et al ${ }^{9}$
Lin et al ${ }^{10}$
Idzelene ${ }^{11}$
Ferrari et al ${ }^{12}$
Maeda et al ${ }^{13}$
Buckton et al ${ }^{14}$
Hansteen et al ${ }^{15}$
Bratkowska et al ${ }^{16}$
Nielsen et al ${ }^{17}$
Subtotal
Overall

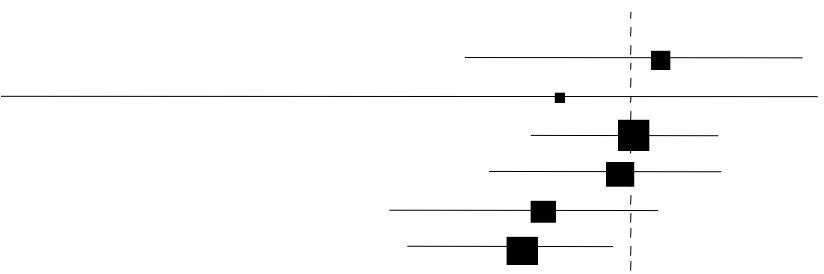

.

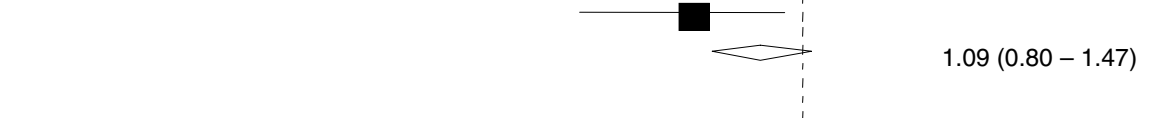




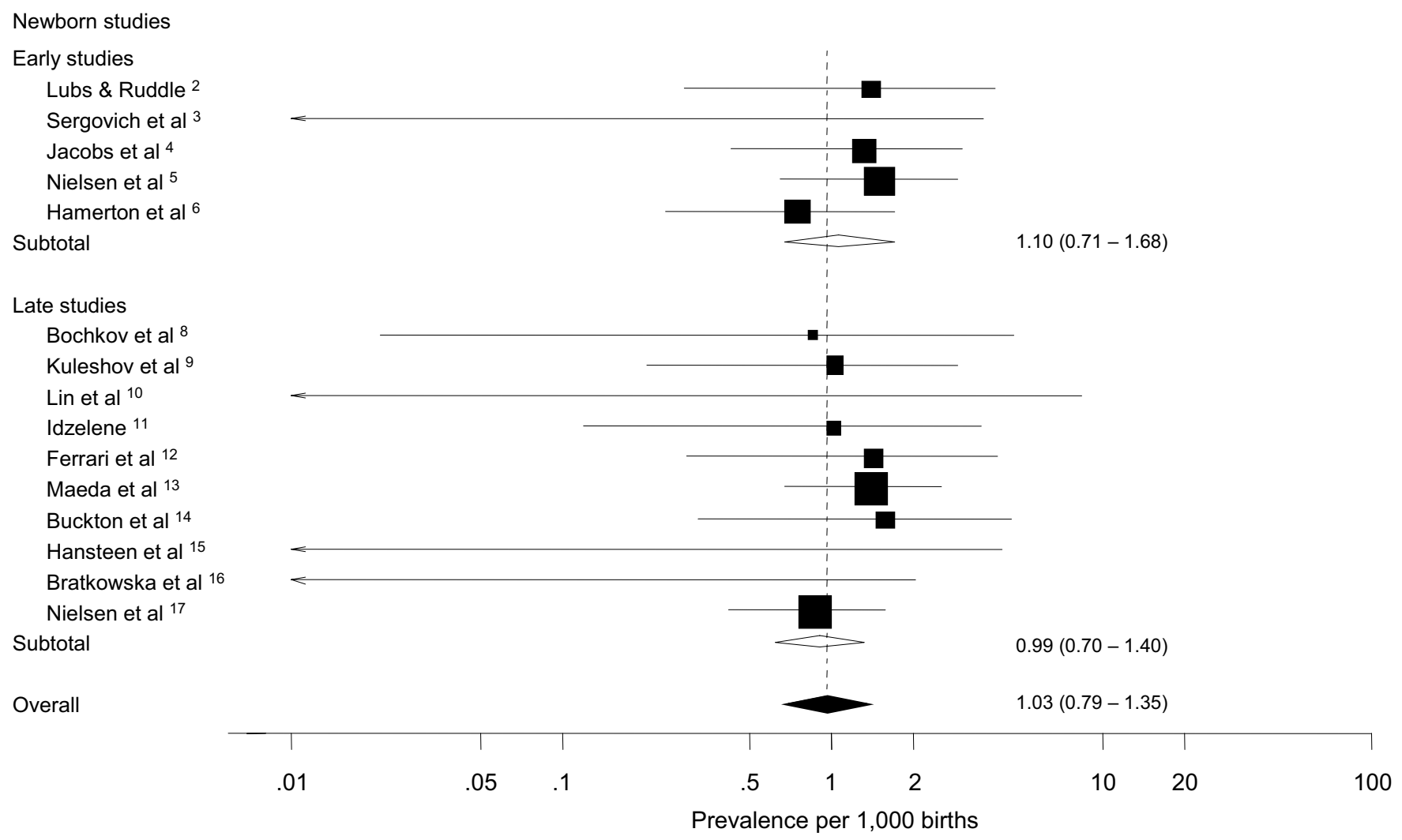

Figure 3 Live birth prevalence of XXX. Squares are estimates of prevalence sized according to the size of the study (studies with no affected babies have no square). Horizontal lines are the $95 \%$ confidence intervals of the prevalence in each study. Empty diamonds are summary estimates of the prevalence for the early and late studies. Full diamond is overall estimate of early and late series combined, with the vertical dashed line at the estimate. The size of the diamond indicates the $95 \% \mathrm{Cl}$.

Table 2 Estimated prevalence of 47,XYY, 47XXY and 47,XXX in recognised pregnancies using studies of newborns, spontaneous abortions and perinatal deaths

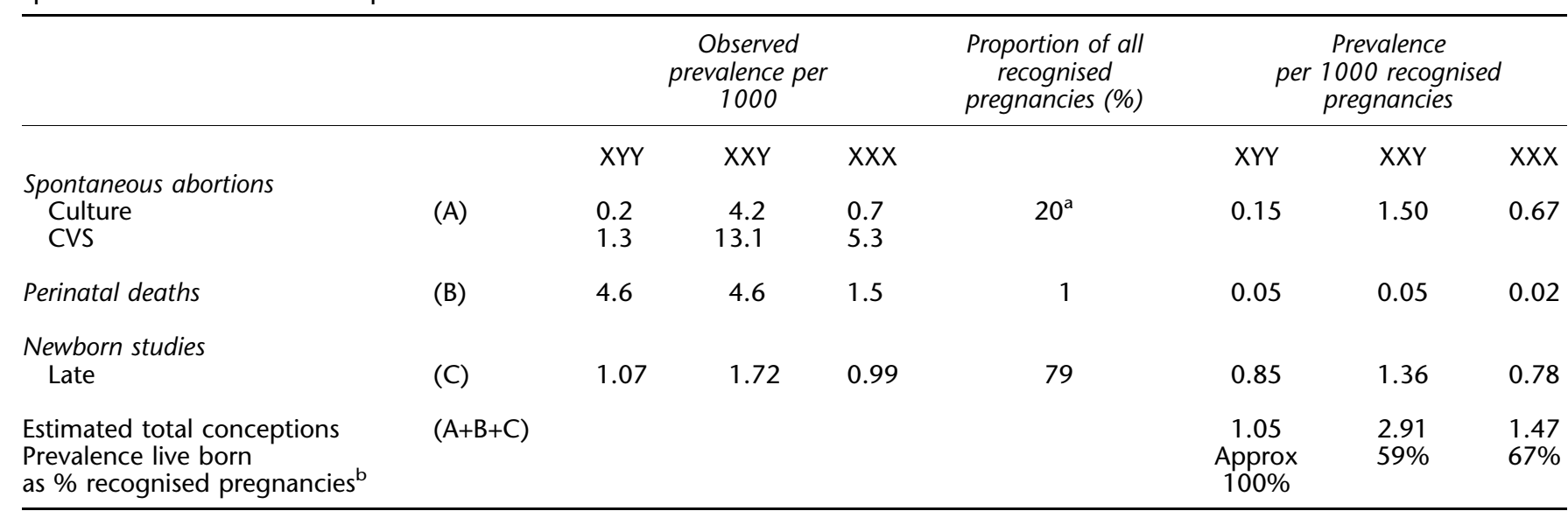

${ }^{a}$ The prevalence per 1000 recognised pregnancies is calculated as $20 \%$ of the mean of the two types of spontaneous abortion series weighted according to the number of sex chromosome trisomies identified.

${ }^{b}$ Prevalence live born, as \% recognised pregnancies is the prevalence of total conceptions/prevalence in newborn studies (for example XXY $=1.36$ / $1.72=59 \%$ ).

from the same factors, presumably environmental, that are associated with the well documented fall in sperm count in our species. ${ }^{44-46}$ There are two observations that lend some to support this suggestion. The frequency of aneuploidy in sperm, studied using FISH technology, (summarised in Hassold 1998) ${ }^{47}$ has shown $1 \%$ of sperm 
to be hyperhaploid in normal males and the sex chromosomes to be the most frequently involved. Secondly, when the frequency of hyperhaploidy in sperm from men with a low sperm count is examined the majority show an increase in disomic sperm, which is especially marked for the sex chromosomes. Martin et l $^{48}$ compared hyperhaploidy in males with mild, moderate and severe oligospermia and found an increase in sex chromosome aneuploidy as the sperm count fell. While there was a significant increase for all three types of sex chromosome aneuploidy, it was much more pronounced in the XY class where it reached a frequency of $0.68 \%$ in the severely oligospermic males. Similar results have been reported in other studies of nondisjunction in low quality semen. ${ }^{49}$

The hypothesis that the increase in XXYs is the result of an increase in paternal, rather than maternal, nondisjunction could in principal be tested by comparing the parental origin of a large series of XXY males born prior to the 1970s with those born more recently. The hypothesis predicts that the proportion of XXYs due to paternal nondisjunction would be substantially higher in the latter than the former group.

\section{Acknowledgements}

We are very grateful to Drs Dorothy Warburton, Terry Hassold, Johannes Neilsen, Alan Wright and Sven Cnattingius for allowing us to use their unpublished data. We are also grateful to all the cytogenetic laboratories contributing their data to CAD. CAD is funded by BDF Newlife (www.bdfnewlife.co.uk), PJ is funded by the Wellcome Trust Grant 038823.

\section{References}

1 Hook EB, Hamerton JL: The frequency of chromosome abnormalities detected in consecutive newborn studies - differences between studies - results by sex and severity of phenotypic involvement; in Porter IH, Hook EB (eds): Population Cytogenetics. New York:National Academy Press, 1977, pp 63-79.

2 Lubs HA, Ruddle F: Application of quantitative karyotypy to chromosome variation in 4400 consecutive newborns; in Jacobs PA, Price WH, Law P (eds): Human Population Cytogenetics. Edinburgh:University of Edinburgh Press (Pfizer Medical Monograph 5), 1970, pp 120-142.

3 Sergovich F, Valentine GH, Chen ATL, Kinch RAH, Smout MS: Chromosome aberrations in 2159 consecutive newborn babies. N Engl J Med 1969; 280: 851-855.

4 Jacobs PA, Melville M, Ratcliffe S: A cytogenetic survey of 11680 newborn infants. Ann Hum Genet 1974; 37: 359-376.

5 Nielsen J, Wohlert M, Faaborg-Andersen J et al: Incidence of chromosome abnormalities in newborn children. Comparison between incidences in 1969-1974 and 1980-1982 in the same area. Hum Genet 1982; 61: 98-101.

6 Hamerton JL, Canning N, Ray M, Smith S: A cytogenetic survey of 14069 newborn infants. Clin Genet 1975; 8: 223-243.

7 Walzer S, Gerald PS: A chromosome survey of 13751 male newborns; in Porter JH, Hook EB (eds): Population Cytogenetics. New York:Academic Press New York, 1977, pp 45-61.

8 Bochkov NP, Kuleshov NP, Chebotarev AN, Alekhin VI, Midian SA: Population cytogenetic investigation of newborns in Moscow. Humangenetick 1974; 22: 139-152.
9 Kuleshov NP, Bochkov NP, Alekhin VI, Debova GA, Platanova VI: Cytogenetic study of 6000 newborn infants. Genetika 1978; 14: 340-347.

10 Lin CC, Godeon M, Griffith P et al: Chromosome analysis on 930 consecutive newborn children using quinacrine fluorescent banding technique. Hum Genet 1976; 31: 315-328.

11 Idzelene IP: Chromosome anomalies among newborn children in Lithuania. Genetika 1978; 14: 2193-2196.

12 Ferrari I, Jorge SM, Toledo V, Michele JL: Cytogenetic study of 4296 consecutive newborns at the University Hospital of Ribeirao Preto, Sao Paulo, Brazil. Rev Brasil Genet 1982; 3: 631-637.

13 Maeda T, Ohno M, Matsunobu A, Yoshihara K, Yabe N: A cytogenetic survey of 14835 consecutive liveborns. Ipn J Hum Genet 1991; 36: 117-129.

14 Buckton KE, O'Riordan ML, Ratcliffe S, Slight J, Mitchell M, McBeath S: A G-band study of chromosomes in liveborn infants. Ann Hum Genet 1980; 43: 227-230.

15 Hansteen I-L, Varslot K, Steen-Johnsen J, Langard S: Cytogenetic screening of a new-born population. Clin Genet 1982; 21: 309-314.

16 Bratkowska W, Hubner H, Ferenc T, Szpakowski M, Krajewski J, Budzko W: Chromosome studies in 3665 consecutive newborn children. Acta Anthropogenetica 1985; 9: 153-161.

17 Nielsen J, Wohlert M: Chromosome abnormalities found among 34910 newborn children: results from a 13-year incidence study in Århus, Denmark. Hum Genet 1991; 87: 81-83.

18 Lauritsen JG: Aetiology of spontaneous abortion. A cytogenetic and epidemiological study of 288 abortuses and their parents. Acta Obstet Gynecol Scand 1976; (Suppl 52): 1-29.

19 Kajii T, Ferrier a, Niikawa N, Takahara H, Ohama K, Avirachan S: Anatomic and chromosomal anomalies in 639 spontaneous abortuses. Hum Genet 1980; 55: 87-98.

20 Warburton D, Byrne J, Canki N: Chromosome Abnormalities and Prenatal Development: an Atlas. New York:Oxford University Press, 1991.

21 Hassold TJ, Chen N, Funkhouser J et al: A cytogenetic study of 1000 spontaneous abortions. Ann Hum Genet 1980; 44: $151-178$.

22 Lin CC, Braekeleer De M, Jambo H: Cytogenetic studies in spontaneous abortions: the Calgary experience. Can Genet Cytol 1985; 27: $565-570$.

23 Nagaishi M, Yamamoto T, Iinuma K, Shimomura K, Berend SA, Knops J: Chromosome abnormalities identified in 347 spontaneous abortions collected in Japan. J Obstet Gynaecol Res 2004; 30: $237-241$.

24 Guerneri S, Bettio D, Simoni G, Brambati B, Lanzani A, Fraccaro M: Prevalence and distribution of chromosome abnormalities in a sample of first trimester internal abortions. Hum Reprod 1987; 2: $735-739$.

25 Ohno M, Maeda T, Matsunobu A: A cytogenetic study of spontaneous abortions with direct analysis of chorionic villi. Obstet Gynecol 1991; 77: 394-398.

26 Sánchez JM, Franzi L, Collia F, De Diaz SL, Panal M, Dubner M: Cytogenetic study of spontaneous abortions by transabdominal villus sampling and direct analysis of villi. Prenat Diagn 1999; 19: 601-603.

27 Eiben B, Bartels I, Bahr-Porsch S et al: Cytogenetic analysis of 750 spontaneous abortions with the direct-preparation method of chorionic villi for studying genetic causes of pregnancy wastage. Am J Hum Genet 1990; 47: 656-663.

28 Ljunger E, Cnattingius S, Lundin C, Annerén G: Chromosomal anomalies in first-trimester miscarriages. Acta Obstet Gynecol Scand 2005; 84: 1103-1107.

29 Strom CM, Ginsberg N, Applebaum M et al: Analyses of 95 first-trimester abortions by chorionic villus sampling and karyotype. J Assist Reprod Genet 1992; 9: 458-461.

30 Machin GA, Crolla JA: Chromosome constitution of 500 infants dying during the perinatal period. With an appendix concerning other genetic disorders among these infants. Humangenetik 1974; 23: $183-198$ 
31 Kuleshov NP: Chromosome anomalies of infants dying during the perinatal period and premature newborn. Hum Genet 1976; 31: $151-160$.

32 Sutherland GR, Carter RF, Bauld R, Smith II, Bain AD: Chromosome studies at the paediatric necropsy. Ann Hum Genet 1978; 42: $173-181$.

33 Ferguson-Smith MA, Yates JRW: Maternal age specific rates for chromosome aberrations and factors influencing them: report of a collaborative European study on 52965 amniocenteses. Prenat Diagn 1984; 4: 5-44.

34 Schreinemachers DM, Cross PK, Hook EB: Rates of trisomies 21, 18,13 and other chromosome abnormalities in about 20000 prenatal studies compared with estimated rates in live births. Hum Genet 1982; 61: 318-324.

35 Scott C: The chromosome abnormality database - the development of a new internet search facility www.ukcad.org.uk. J Med Genet 2004; 41: S59.

36 Ryall RG, Callen D, Cocciolone $\mathrm{R}$ et al: Karyotypes found in the population declared at increased risk of Down syndrome following maternal serum screening. Prenat Diagn 2001; 21: $553-557$.

37 Spencer K, Tui N, Nicolaides K: Maternal serum free beta-hCG and PAPP-A in fetal sex chromosome defects in the first trimester. Prenat Diagn 2000; 20: 390-394.

38 Hassold T: Mosaic trisomies in human spontaneous abortions. Hum Genet 1982; 61: 31-35.

39 Thomas NS, Collins AR, Hassold TJ, Jacobs PA: A reinvestigation of non-disjunction resulting in 47 , XXY males of paternal origin. Eur J Hum Genet 2000; 8: 805-808.
40 Thomas NS, Ennis S, Sharp AJ et al: Maternal sex chromosome non-disjunction: evidence for $\mathrm{X}$ chromosome-specific risk factors. Hum Mol Genet 2001; 10: 243-250.

41 May KM, Jacobs PA, Lee M et al: The parental origin of the extra X chromosome in 47, XXX females. Am J Hum Genet 1990; 46: 754-761.

42 Robinson DO, Jacobs PA: The origin of the extra Y chromosome in males with a 47, XYY karyotype. Hum Mol Genet 1999; 8: 2205-2209.

43 Hunt PA, Hassold TJ: Sex matters in meiosis. Science 2002; 296 $2181-2183$

44 Andersen AG, Jensen TK, Carlsen E et al: High frequency of suboptimal semen quality in an unselected population of young men. Hum Reprod 2000; 15: 366-372.

45 Irvine S, Cawood E, Richardson D, MacDonald E, Aitken J: Evidence of deteriorating semen quality in the United Kingdom: birth cohort study in 577 men in Scotland over 11 years. BM) 1996; 312: $467-471$.

46 Carlsen E, Giwercman A, Keiding N, Skakkebaek NE: Evidence for decreasing quality of semen during past 50 years. BMJ 1992; 305: 609-612.

47 Hassold TJ: Nondisjunction in the human male. Curr Top Dev Biol 1998; 37: 383-407.

48 Martin RH, Rademaker AW, Greene C et al: A comparison of the frequency of sperm chromosome abnormalities in men with mild, moderate, and severe oligozoospermia. Biol Reprod 2003; 69: $535-539$

49 Finkelstein S, Mukamel E, Yavetz H, Paz G, Avivi L: Increased rate of nondisjunction in sex cells derived from low-quality semen. Hum Genet 1998; 102: 129-137.

Supplementary Information accompanies the paper on European Journal of Human Genetics website (http://www.nature.com/ejhg) 